\title{
Nucleation and Growth of a Flux Instability in Superconducting $\mathrm{YBa}_{2} \mathrm{Cu}_{3} \mathrm{O}_{7}-x$ Films
}

\author{
P. Leiderer, J. Boneberg, P. Brüll, V. Bujok, and S. Herminghaus \\ Fakultät für Physik, Universität Konstanz, 7750 Konstanz, Germany
}

(Received 24 June 1993)

\begin{abstract}
Using a high speed magneto-optic technique we have investigated the dynamics of a new flux instability in thin superconducting $\mathrm{YBa}_{2} \mathrm{Cu}_{3} \mathrm{O}_{7-x}$ films exposed to an external magnetic field of several $10^{-2} \mathrm{~T}$. The instability was nucleated by a 5 ns laser pulse, which heated a small spot of the sample in a region of high shielding currents. Two subsequent regimes in the development of the instability are discovered, which give rise to strikingly different flux distributions. The formation of flux branches, which are characteristic for the second stage, occurs on a time scale of a hundred nanoseconds.
\end{abstract}

PACS numbers: $74.60 . \mathrm{Ge}, 74.60 . \mathrm{Jg}$

Flux dynamics in superconductors is a long-standing topic, which is still of considerable current interest [1]. Since the penetration of magnetic flux is of fundamental importance for the superconducting properties of a sample, it has been studied in numerous investigations, in particular in conventional type II superconductors [2]. The motion of flux lines in these materials depends crucially on the presence of pinning centers, which lead to flux gradients inside the superconductors as described, e.g., by the Bean model. Since these field distributions do not correspond to an equilibrium distribution of the flux line lattice, the system can eventually become unstable, and flux enters the sample in momentary events in the form of "flux jumps" [3,4]. For massive samples on a millimeter scale the characteristic time for the redistribution of flux following such an instability has been found to be in the range of milliseconds [4].

In this Letter we report on the dynamics of propagating magnetic flux fronts resulting from an instability in thin films of the type II superconductor $\mathrm{YBa}_{2} \mathrm{Cu}_{3} \mathrm{O}_{7-x}$. In contrast to most earlier studies of flux jumps, we have artificially triggered the instability by a local perturbation of the superconducting state, using a focused laser pulse. The measurements not only yield propagation speeds of the flux fronts 5 orders of magnitude larger than for massive samples, but also show for the first time two strikingly different regimes of pattern formation in the flux distribution.

Our $\mathrm{YBa}_{2} \mathrm{Cu}_{3} \mathrm{O}_{7-x}$ samples were epitaxial $c$-oriented films with a thickness of $300 \mathrm{~nm}$, deposited on $1 \times 1 \mathrm{~cm}^{2}$ $\mathrm{LaAlO}_{3}$ substrates by laser ablation [5]. The measurements were carried out in an optical cryostat equipped with a superconducting magnet. The direction of the magnetic field was perpendicular to the sample plane. In order to detect the flux distribution in the sample we used a reflection magneto-optic technique, with a EuS film evaporated onto a glass plate as the active element [6,7]. An additional $100 \mathrm{~nm}$ thick Al layer covering the back side of the detector film improved the reflectivity [7]. The whole stack was spring loaded against the sample in order to keep the gap between the superconductor and the EuS/Al film down to the order of a $\mu \mathrm{m}$.

The sample in general was zero field cooled and during the measurement was immersed in superfluid ${ }^{4} \mathrm{He}$ at 1.8 K. As a magnetic field was applied, the evolution of the flux distribution in the sample was recorded with a video camera using a standard magneto-optic setup and a 3 $\mathrm{mW} \mathrm{He}-\mathrm{Ne}$ laser (wavelength $\lambda=633 \mathrm{~nm}$ ) as a light source, at a spatial resolution better than $10 \mu \mathrm{m}$. The field was typically increased up to a few $10^{-2} \mathrm{~T}$, where flux entering from the sample edges roughly filled several ten percent of the film area, leaving a large portion of the sample in the Meissner state [7].

In order to perturb this state and to initiate the magnetic instability, a pulse of a frequency doubled Nd:YAG laser $(\lambda=532 \mathrm{~nm}$, FWHM $7 \mathrm{~ns}$, energy up to $5 \mathrm{~mJ})$ was focused onto the superconducting film from the back side through the (polished) $\mathrm{LaAlO}_{3}$ substrate. The laser focus with a diameter of $50 \mu \mathrm{m}$ could be positioned deliberately on the sample surface, so that the nucleation of the instability could be started from various spots of the film. At the laser energies used here the sample temperature in the focus, although not measured directly, supposedly rose above the critical temperature of the superconductor $\left(T_{c}=92 \mathrm{~K}\right)$.

As it turned out in the course of the experiments a rather high temporal resolution was required to resolve the rapid development of the flux jumps in our films. A double pulse technique was applied to achieve this goal [6]. Part of the Nd:YAG beam was separated by a beam splitter, passed through a variable delay line of several 10 $\mathrm{ns}$, and frequency shifted to $\lambda=635 \mathrm{~nm}$ (details are described in Ref. [6]). This pulse was fed into the magneto-optic light path to illuminate the EuS film at a well-defined time delay after the instability had been triggered, and thus provided a momentary snapshot of the flux distribution which was also recorded by the video camera. The cw He-Ne source was blocked for a few video frames before and after the laser pulse.

We first present in Fig. 1 examples for the steady state patterns of a $\mathrm{YBa}_{2} \mathrm{Cu}_{3} \mathrm{O}_{7-x}$ film in a constant external field, $B_{\text {ext }}=60 \mathrm{mT}$ in this case. Figure 1 (a), taken before the Nd:YAG shot, indicates some penetration of flux at the sample edges (white regions), whereas the central, dark area represents the Meissner phase. Its overall shape is a typical field penetration pattern as shown be- 


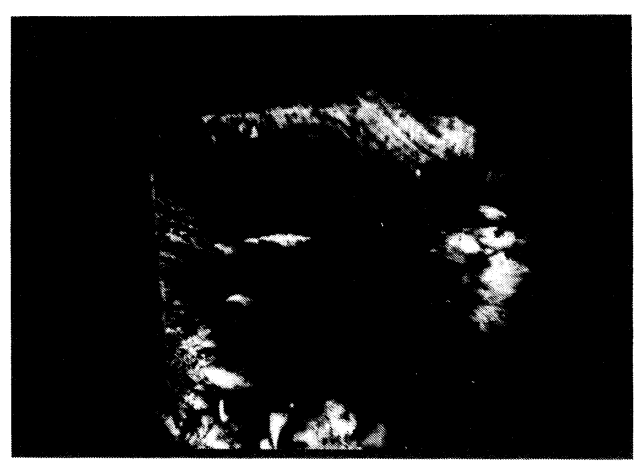

ure 1(b) shows the same film after laser heating a spot approximately at the sample center. Although somewhat difficult to discern, a rather large portion of the former Meissner region now displays an increased, nearly constant light intensity - and hence the presence of a perpendicular magnetic field-indicating that the laser pulse has nucleated a large scale redistribution of magnetic flux.

Next we started again from a field distribution as depicted in Fig. 1(a)-after heating the sample above $T_{c}$, zero field cooling and applying $B_{\text {ext }}=60 \mathrm{mT}$ - but now the laser focus was adjusted near the sample edge, close to a "weak spot" where flux had already entered the film. Although the overall contour of the resulting laserinduced flux distribution, as seen in Fig. 1(c), is similar to the one in Fig. 1(b), the fine structure is obviously completely different. Instead of the more or less spatially constant flux density in Fig. 1(b) one now observes pronounced, branchlike structures with an average spacing of $0.3 \mathrm{~mm}$ and a width of the branches of $0.1 \mathrm{~mm}$. On closer inspection one can also distinguish in Fig. 1(c) an area of about $0.5 \mathrm{~mm}$ radius around the nucleation spot with a structureless flux distribution without branches. This is a first hint that flux motion triggered by the laser pulse actually develops in two steps [10]: (i) First, a perturbation grows in the Meissner phase around the nucleation spot in a roughly isotropic way, forming a region where flux, accumulated at some weak parts of the film before, is afterwards distributed homogeneously within the perturbed area; (ii) as soon as this region makes contact with the surrounding externally applied field at some point, massive avalanchelike penetration of external flux sets in, and branches start to spread out from the rim of the homogeneous area.

More conclusive evidence for the hypothesis of such a two-step process comes from the time-resolved measurements to be discussed now. Figure 2 displays a sequence of pictures taken before ( $2 a), 56 \mathrm{~ns}$ after ( $2 \mathrm{~b})$, and a few seconds after (2c) the Nd:YAG pulse. Figure 2(b) thus represents a transient state, whereas 2 (a) and 2 (c) are steady distributions. A comparison of Figs. 2(b) and 2(c) shows that already after $t=56 \mathrm{~ns}$ the flux structure has nearly reached its final size. Nevertheless, some of the branches are not yet fully developed and apparently still are to grow a few tenths of a mm. A series of such recordings with variable delay times has been used to investigate the growth dynamics of the flux structures in more detail. These studies reveal that the spatially homogeneous field distributions like in Fig. 1(b) develop extremely fast on a time scale which is not even accessible with our temporal resolution of $10 \mathrm{~ns}$. We therefore can only estimate that the speed at which the front of this phase propagates is larger than $2 \times 10^{5} \mathrm{~m} / \mathrm{s}$. The propagation speed of the branches, on the other hand, was small enough that it could be determined with our technique. In Fig. 3 we have plotted results for the mean velocity, $\langle v\rangle=l / \tau$, where $l$ is the length of the flux branches 


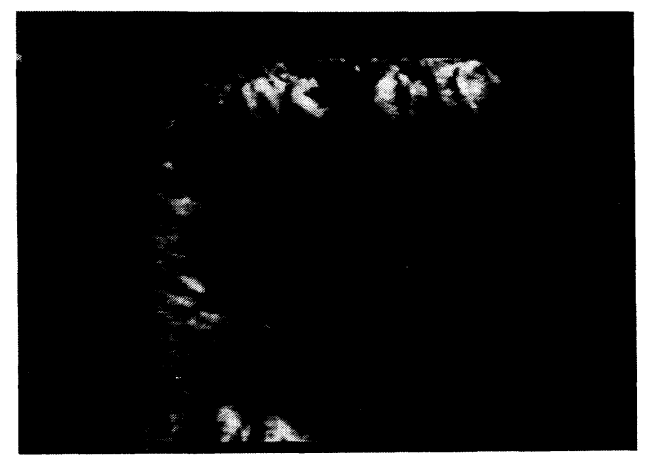

(a)

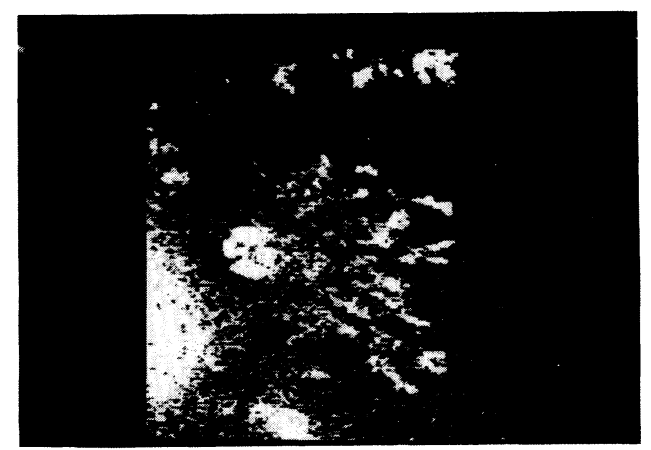

(b)

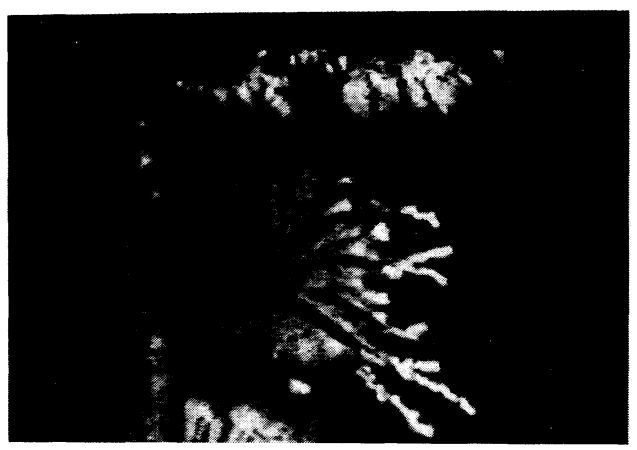

(c)

FIG. 2. Time evolution of the instability in the magnetic field distribution. The frames correspond to a $4 \times 4 \mathrm{~mm}^{2}$ section of the superconductor $\left(T=1.8 \mathrm{~K}, B_{\mathrm{ext}}=25 \mathrm{mT}\right)$. (a) Before the lasser pulse, (b) $56 \mathrm{~ns}$ after the laser pulse, and (c) final flux distribution. The width of the branches is similar as in Fig. 1(c) $(\approx 100 \mu \mathrm{m})$.

and $\tau$ is the delay time. The data yield $\langle v\rangle=(5 \pm 2)$ $\times 10^{4} \mathrm{~m} / \mathrm{s}$, which, for comparison, is an order of magnitude higher than the velocity of sound in $\mathrm{YBa}_{2} \mathrm{Cu}_{3} \mathrm{O}_{7-x}$ [11].

We now turn to possible interpretations of the observed phenomenon. As a superconducting spot carrying large shielding currents is heated up to temperatures where $j_{c}$ has dropped considerably or is even zero, dissipation and a redistribution of the shielding currents will take place. We concentrate here on the second step of the instability,

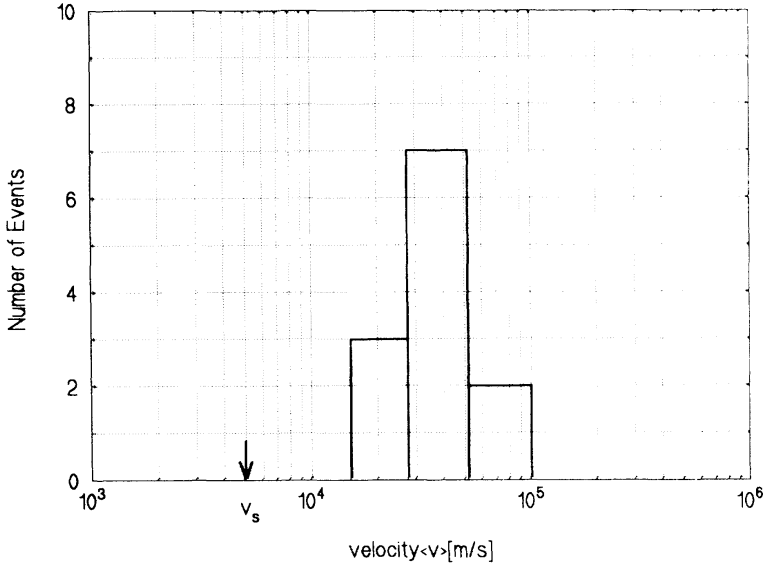

FIG. 3. Histogram of the average spreading velocity $\langle v\rangle$ of flux branches, derived from several recordings [as in Fig. 2(b)] at different delay times $\tau$. The quoted values were obtained from $\langle v\rangle=l / \tau$ (where $l$ is the length of the branch pattern at this time $\tau$ ), and therefore only represent a lower limit for the actual spreading velocity. The arrow marks the velocity of sound $v_{s}$ in $\mathrm{YBa}_{2} \mathrm{Cu}_{3} \mathrm{O}_{7-x}[11]$.

where more experimental information is available. Regarding the high propagation speed of the flux branches, a theoretical description of the phenomenon has to rest on the interaction of the supercurrents with the electromagnetic field, in contrast to theories based on primarily thermal quench fronts $[2,12]$.

Our approach is similar to the one used by Pippard [13] to study the dynamics of the quench front in bulk superconductors. We start combining Maxwell's and London's equations to the equation of motion,

$$
\left(\Delta-\partial_{t t} / c^{2}-l / \lambda^{2}-\mu \sigma \partial_{t}\right) \Phi=0,
$$

where $\Delta$ is the Laplace operator, $\partial_{t}$ indicates the second derivative with respect to time, $\lambda$ is the London penetration depth, $\mu$ the magnetic permeability, and $\sigma$ the normal conductivity of the material. $\Phi$ stands for either the electric or the magnetic field. The field equations for the normal conducting state are obtained by setting $l / \lambda^{2}=0$. As a simple model, we consider a straight quench front moving with the velocity $v$. A straightforward calculation yields the decay lengths $l$ of the fields into the superconducting $\left(l_{\mathrm{sc}}\right)$ and into the normal region $\left(l_{\mathrm{nc}}\right)$. We obtain $l_{\mathrm{sc}} \approx \lambda$ and $l_{\mathrm{nc}} \approx \sqrt{R d}$, where $R$ is the lateral sample dimension and $d$ the sample thickness. Inserting typical sample dimensions yields $l_{\mathrm{nc}} \approx 40 \mu \mathrm{m}$. Thus the minimum width of any structure in the normal region must be at least $2 l_{\mathrm{nc}} \approx 80 \mu \mathrm{m}$ which agrees very well with the observed branch width of $100 \mu \mathrm{m}$. In order to shed light on the formation mechanism of the branches we have also carried out a linear stability analysis of a straight quench front [14]. As the calculations show, the front is stable with respect to small perturbations, as in the case of bulk superconductors [15]. This suggests that the branches do 
not evolve from a soft mode of the straight front. In fact, it seems that the branch number grows during the whole quench process by repeated splitting of individual branches, in contrast to a soft mode mechanism which would produce a large number of evenly spaced branches from the start. Apparently, the rather uniform spacing in Fig. 1(c) originates from the statistics of the splitting process. For a quantitative prediction of the front (or growth) velocity, detailed information about the magnetic field distribution would be required [13]. In addition, eddy currents in the aluminum mirror adjacent to the superconducting film would have to be taken into account [4].

In summary, we have studied the evolution of a novel instability nucleated by a laser pulse in thin superconducting films exposed to a static magnetic field. We find two regimes: first, a process where the perturbation leads to a homogeneous redistribution of flux over a certain part of the sample on a time scale less than $10 \mathrm{~ns}$, and a second step, where flux entering the sample from the outside penetrates the Meissner phase in the form of branches which propagate at a speed of about $5 \times 10^{4}$ $\mathrm{m} / \mathrm{s}$. Some of the characteristics of this instability follows directly from a combination of Maxwell's and London's equations; others, like the origin of the branching, require further investigations. The experiments have been performed with a high- $T_{c}$ superconductor, but we expect similar phenomena to occur also for conventional hard type II superconductors.

We acknowledge helpful discussions with A. L. Rakhmanov and E. H. Brandt and thank B. Stritzker for supplying the superconducting sample. This work was supported by Bundesministerium für Forschung und
Technologie, Grant No. 13N5705 and by the Schwerpunktprogramm Land Baden-Württemberg.

[1] M. R. Freeman, Phys. Rev. Lett. 69, 1691 (1992).

[2] See, e.g., R. G. Mints and A. L. Rakhmanov, Rev. Mod. Phys. 53, 551 (1981); R. P. Huebener, Magnetic Flux Structures in Superconductors (Springer-Verlag, Berlin, 1979).

[3] R. B. Harrison, L. S. Wright, and M. R. Wertheimer, Phys. Rev. B 7, 1864 (1973).

[4] R. B. Harrison, L. S. Wright, and M. R. Wertheimer, J. Appl. Phys. 45, 403 (1974); R. B. Harrison, J. P. Pendrys, and L. S. Wright, J. Low Temp. Phys. 18, 113 (1975).

[5] J. Fröhlingsdorf, W. Zander, and B. Stritzker, Solid State Commun. 67, 965 (1988).

[6] V. Bujok, P. Brüll, J. Boneberg, S. Herminghaus, and P. Leiderer, Appl. Phys. Lett. 63, 412 (1993).

[7] P. Brüll, D. Kirchgässner, and P. Leiderer, Physica (Amsterdam) 182C, 339 (1991).

[8] H. Theuss, A. Forkl, and H. Kronmüller, Physica (Amsterdam) 190C, 345 (1992).

[9] D. Kirchgässner, P. Brüll, and P. Leiderer, Physica (Amsterdam) 195C, 157 (1992).

[10] P. Brüll, Ph.D. thesis, University of Konstanz, Germany, 1992.

[11] T. J. Kim, J. Kowalewski, W. Assmus, and W. Grill, Z. Phys. B 78, 207 (1990).

[12] M. I. Flik and C. L. Tien, J. Heat Transfer 112, 10 (1990).

[13] A. B. Pippard, Philos. Mag. 41, 243 (1950).

[14] V. Bujok, P. Brüll, J. Boneberg, S. Herminghaus, and P. Leiderer, Europhys. Conf. Abstr. 17A, 1023 (1993); S. Herminghaus et al. (to be published).

[15] H. Frahm, S. Ullah, and A. T. Dorsey, Phys. Rev. Lett. 66, 3067 (1991). 


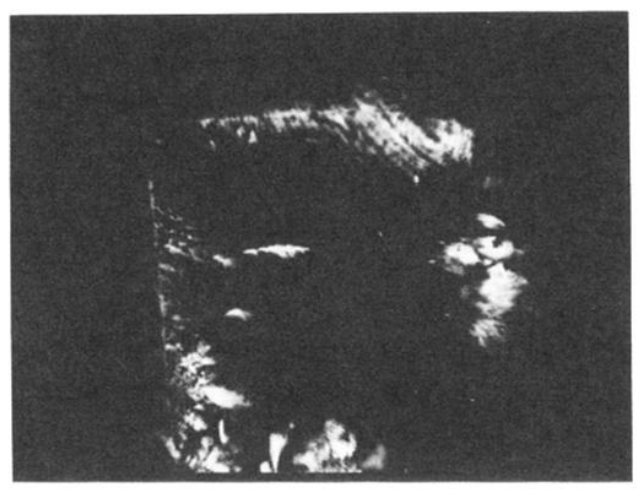

(a)

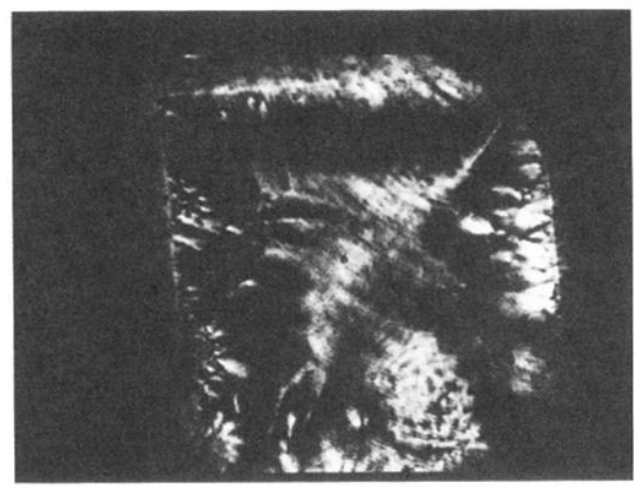

(b)

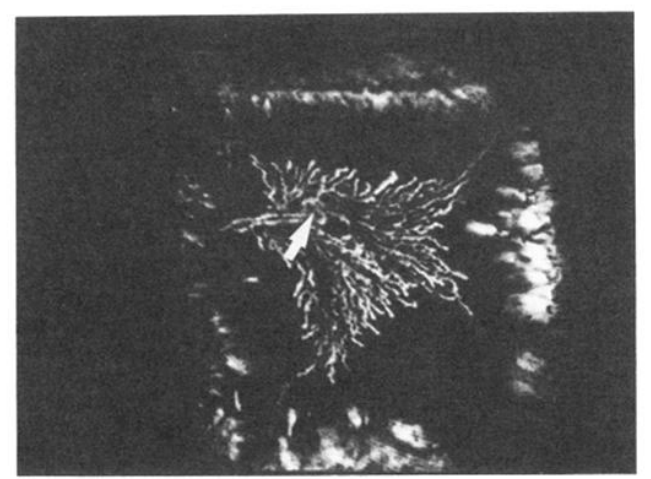

(c)

FIG. 1. Laser-induced changes in the magnetic flux distribution of a thin $\mathrm{YBa}_{2} \mathrm{Cu}_{3} \mathrm{O}_{7-x}$ film. Bright regions correspond to the Shubnikov phase and dark regions to the Meissner phase. The sample size is $1 \times 1 \mathrm{~cm}^{2}$. (a) Flux distribution before the laser pulse $\left(T=1.8 \mathrm{~K}, B_{\text {ext }}=60 \mathrm{mT}\right)$. A major part of the sample is in the Meissner phase. (b) Change of the flux distribution after laser heating a spot in the sample center. (c) Change of the flux distribution after laser heating a spot (position indicated by the white arrow) close to a weak region, where some flux had already penetrated. 


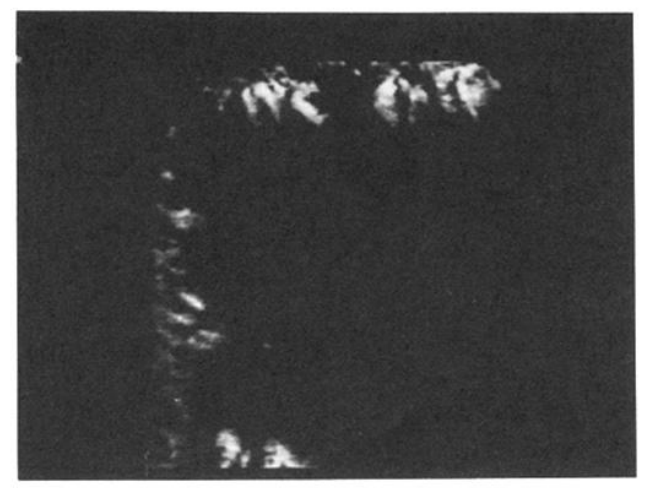

(a)

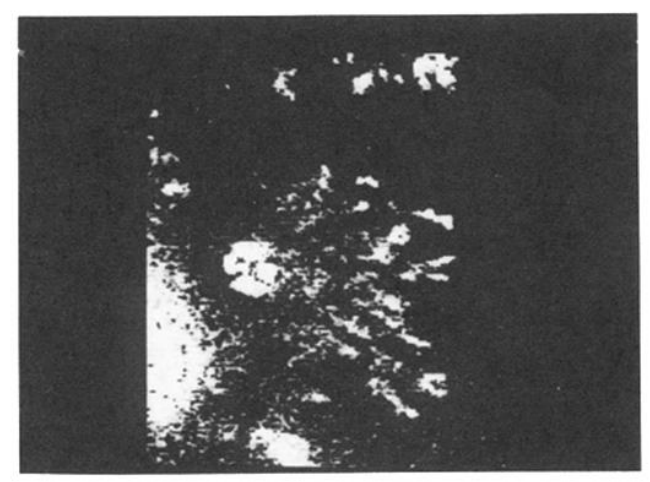

(b)

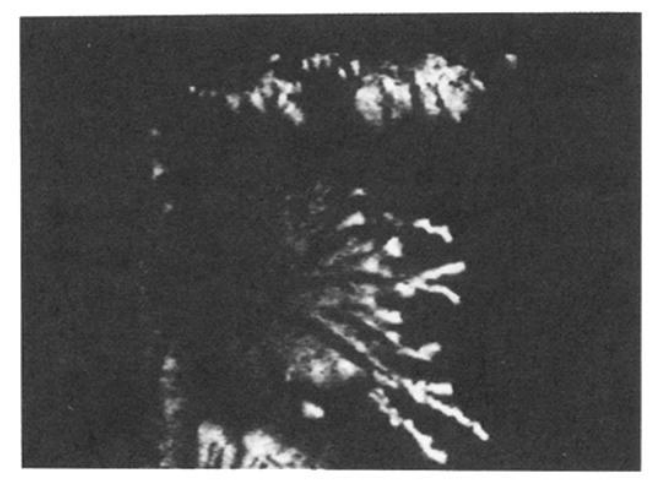

(c)

FIG. 2. Time evolution of the instability in the magnetic field distribution. The frames correspond to a $4 \times 4 \mathrm{~mm}^{2}$ section of the superconductor $\left(T=1.8 \mathrm{~K}, B_{\text {ext }}=25 \mathrm{mT}\right)$. (a) Before the lasser pulse, (b) $56 \mathrm{~ns}$ after the laser pulse, and (c) final flux distribution. The width of the branches is similar as in Fig. 1(c) $(\approx 100 \mu \mathrm{m})$. 